\title{
Gründungsdokument des wissenschaftlichen Sozialismus
}

\section{Engels' Die Lage der arbeitenden Klasse in England als praktisch eingreifende verbindende Erzählung}

Zusammenfassung: Der Beitrag analysiert das Werk von Friedrich Engels »Die Lage der arbeitenden Klasse in England « von 1845 als Gründungsschrift des wissenschaftlichen Sozialismus. Es wird auf die Bedeutung dieses Werkes als strategisch orientierendes Narrativ auf der Grundlage einer sozialwissenschaftlichen Feldstudie und umfassenden empirischen Forschung eingegangen, das auf die Verbindung von Arbeiter*innenbewegung und Sozialismus orientiert und die Notwendigkeit und Unvermeidlichkeit einer kommunistischen sozialen Revolution aus der Geschichte und Struktur der kapitalistischen Gesellschaft sowie dem Emanzipationskampf der Arbeiter*innen ableitet.

Schlagwörter: Marxismus, Sozialismus, Geschichte der Arbeiter*innenbewegung, Kapitalismus

\section{Founding document of scientific socialism \\ Engels' The Condition of the Working Class in England as a practical, intervening unifying narrative}

Abstract: The article analyses the work of Friedrich Engels »The Condition of the Working Class in England «, written in 1845, as the founding work of scientific socialism. The significance of this work as a strategically orienting narrative based on a profound field study and comprehensive empirical research is discussed. It is stressed that the work of Engels focuses on the connection between the workers' movement and socialism and derives the necessity and inevitability of a communist social revolution from the history and structure of the capitalist society and the workers' emancipation struggle.

Keywords: Marxism, socialism, history of the labour movement, capitalism

* Michael Brie ist Vorsitzender des Wissenschaftlichen Beirats der Rosa-Luxemburg-Stiftung. 
E s gibt viele Gründe, an Friedrich Engels zu erinnern, der vor 200 Jahren, am 28. November 1820, in Barmen, einem Gründungsort der modernen deutschen Textilindustrie, geboren wurde. Demokrat und Kommunist, Adjutant in der badischen Revolutionsarmee 1849, Journalist, Schriftsteller, kaufmännischer Angestellter und Teilhaber eines kapitalistischen Industriebetriebs in England, Patriot und Internationalist, Polyglott und Autodidakt mit dem Hang zum Universalgelehrten, aktiver Politiker der Ersten Internationale, Ratgeber der Zweiten Internationale und erster Herausgeber nachgelassener Werke von Karl Marx, Begründer des Marxismus und Lehrer der ersten Generation von Marxist*innen - die Liste ließe sich lange fortsetzen. Er war ein Ausnahmetalent und hat eine weltgeschichtliche Bewegung geprägt. ${ }^{1}$

Den Grund dafür kann man finden, wenn man sein im Frühjahr 1845 erschienenes Buch Die Lage der arbeitenden Klasse in England studiert. Er hat es nach einem fast zweijährigen Aufenthalt in Manchester geschrieben, wo er als Assistent des Geschäftsführers der Firma Ermen \& Engels gearbeitet hatte. Es ist ein Jugendwerk. Engels war gerade 24 Jahre alt. Das Buch ist in gebündelter Gestalt der wichtigste Ertrag seines Aufenthalts in England, auch wenn viele andere Schriften zu nennen wären. ${ }^{2}$ Engels entschlüsselte England als

1 Die Aktualität von Engels wird auch daran deutlich, dass eine ganze Reihe von neueren Publikationen erschienen sind (Carver 2020; Krätke 2020; Lucas u.a. 2020; darunter auch ein Roman: Röhrig 2019).

2 Siehe zu der Gesamtheit der intellektuellen Leistungen Engels' im Zusammenhang
Klassengesellschaft des kapitalistischen Industriezeitalters. Indem er mit den Instrumenten der Struktur- und Klassenanalyse, durch Auswertung vieler Berichte über die Situation in den Fabriken und in den Städten, vor allem aber durch teilnehmende Beobachtung deutlich machte, dass es sich um modernes, auf der Basis der Explosion des Reichtums geschaffenen Elends, der Ungleichheit und Unsicherheit handelt, ist Engels Analyse klassisch und brennend aktuell. Die Corona-Pandemie hat in wenigen Monaten alle Illusionen weggefegt, wir würden nicht in einer Klassengesellschaft leben und Seuchen seien ein Gleichmacher. Was Engels damals dokumentierte, dass Klassenlage, Arbeitsverhältnisse, Wohnsituation, Gesundheit, Lebenserwartung untrennbar zusammengehören, ist auch für die Gegenwart, 180 Jahre später, wieder unübersehbar. Die Verhältnisse beim Fleischverarbeitungsunternehmen Tönnies oder auf in den Unterkünften der Wanderarbeiter*innen der deutschen Agrarwirtschaft sind nur die Spitze des Eisbergs. Es lohnt sich auch deshalb, Engels' Werk erneut in die Hand zu nehmen.

Die Schrift von 1845 wurde in Deutschland unmittelbar nach ihrem Erscheinen vielfach besprochen und in einzelnen Teilen nachgedruckt. Auch eine zweite Auflage erfolgte. Die Vertreter der preußischen Zensur schätzten das Werk als »das beste [...], welches bis heute in deutscher Sprache über die Lage der arbeitenden Klasse in England

mit seinem Manchester-Aufenthalt Brie (2020); dort wird auch auf weitere Literatur verwiesen. 
erschienen ist « (zit. n. Ullrich 1966: 256). Die regierungsnahe Allgemeine Preußische Zeitung beschäftigte sich in einem langen Artikel mit dem Buch. Dort hieß es, dass man "noch kein so übersichtliches und, was das Faktische betrifft, so reichhaltiges Buch über diesen Gegenstand zu Gesicht bekommen « habe und dass »die Geschichte des englischen Proletariats [...] das Lehrbuch der praktischen Erfahrungen « sei, »wie die Entwicklung der Not und der Erniedrigung der armen Bevölkerung, deren Grundursachen auch in Deutschland im Keime vorhanden sind, vor sich « gehe (zit. n. ebd.: 256). Der Marburger Professor der Staatswissenschaften Bruno Hildebrand, einer der Begründer der Historischen Schule der Nationalökonomie in Deutschland, bezeichnete das Buch 1848 sogar "als das kommunistische Evangelium der Tatsachen, worauf sich alle Sozialtheorien berufen können" (zit. n. ebd.: 258) und widmete ihm in seinem Hauptwerk 80 Seiten! Erst das Manifest der Kommunistischen Partei, aus der gemeinsamen Arbeit mit Marx entstanden, wird Engels' Buch diesen Rang streitig machen können.

Was Engels' Buch Die Lage der arbeitenden Klasse in England so besonders macht, sind nicht die einzelnen Fakten, die er zusammengetragen hat. Vieles ist aus vorliegenden Schriften und Artikeln sowie Statistiken schlicht übernommen. Er ist auch mitnichten der Erste, der aus eigener Beobachtung über die Lage der Arbeiterinnen und Arbeiter im globalen industriellen Zentrum der Welt - untrennbar mit moderner Sklaverei und kolonialer Ausbeutung verknüpft - berichtete. Die Bedeutung Manchesters für das neue Zeitalter hatten Zeitgenossen wie Alexis de Tocqueville, Charles Dickens oder Thomas Carlyle beschrieben. Man sprach von der "shock city of the age «. Wer zu dieser Zeit in Manchester war, war im Zentrum der industriellen Entwicklung und einer völlig ungeregelten Urbanisierung, im Herzen des britischen Sozialismus und der englischen Arbeiter*innenbewegung, am Ort eines harten Klassenkampfes. Mit Einschränkungen gilt, dass nicht die Darstellungen im Buch neu waren (zumindest nicht in England), sondern originär war die organische Verbindung der sozialistischen Orientierung mit einer neuen Anschauung von Gesellschaft und ihren treibenden Akteuren sowie den politischen Perspektiven. Das Werk stellt eine brillante Komposition dar, ein in sich stimmiges Narrativ, sprich: Engels hatte eine neue große Erzählung geschaffen.

Es gibt viele, die an diesem Punkt stocken: Bilden Wahrheit und Erzählung nicht unüberbrückbare Gegensätze? Sind Erzählungen denn mehr als willkürliche Konstruktionen, denen kein objektiver Gehalt zukommt; sind sie nicht bestenfalls Opium derer, die keine wirkliche Macht über ihr Leben haben? Dies ist ein fataler Irrtum. Jeder Diskursbeitrag, der Wahrheit für sich beansprucht, ist zwangsläufig ein Narrativ. Aussagen über reale Verhältnisse werden miteinander verbunden und eingebettet. Nur dadurch werden sie überhaupt verständlich und erhalten eine objektive Bedeutung, können sinnvoll interpretiert werden. Eine solche Erzählung hat notwendiger Weise drei Ebenen: die der Fakten, die ihrer Verbindung zu einem Ganzen und die der Deutung dieses Ganzen. Erzählun- 
gen haben eine ungeheure Bedeutung: Erstens sind Orientierung in einer komplexen Welt und Handlungsfähigkeit an Erzählungen gebunden. Um in »einer modernen Welt zu leben, braucht man [...] eine Theorie der Ereignisse und der Repräsentation von Ereignissen nicht nur, um die Welt zu verstehen, sondern um in ihr zu bestehen und zu überleben « (Puckett 2016: 16, Übers.: M.B.). Wie Bourdieu schrieb, ist zweitens jede Theorie »ein Programm für die Wahrnehmung " (Bourdieu 1990: 132). Theorien sind aber vor allem Erzählungen über die Wirklichkeit mit Wahrheitsanspruch. Neue Erzählungen machen vorher Unsichtbares sichtbar. Drittens konstituieren sich kollektive Akteure vor allem über solche Erzählungen. Ein "Wir« ist nur möglich, wenn gemeinsame Ansichten über Ziele und Mittel, Gemeinsamkeiten und Unterschiede, Bündnispartner und Feinde besteht. Hinter der Losung $»$ Wir sind die 99 Prozent« oder »Black Lives Matter« stehen Erzählungen, die Orientierung und Handlungsfähigkeit herstellen, Wahrnehmung neu ausrichten und Gemeinsamkeit schaffen.

Entstanden ist die Erzählung, die dem Werk Die Lage der arbeitenden Klasse in England zugrunde liegt, aus der Konfrontation des Linkshegelianers Engels, der sich ganz in der Tradition der Jakobiner sah und gerade erst 1842 , so zumindest Moses Heß, von diesem zum Kommunismus »bekehrt« worden war (Heß 1843: 103), mit England im Allgemeinen und Manchester im Besonderen. Engels hatte von $\mathrm{He} ß$ die Deutung übernommen, dass - während Deutschland das Land der philosophischen Revolution und Frankreich das der politischen Revolution sei - England das Land der kommenden großen sozialen (kommunistischen) Revolution werde. Es waren Engels' ausgedehnte Feldstudien vor Ort, sein Eindringen in immer neue Wissensgebiete, vornehmlich die politische ökonomie und englische Geschichte, seine direkte Begegnung mit der englischen sozialistischen und Arbeiter*innenbewegung, seine laufende Berichterstattung über die englische Politik, Gesellschaft und Kultur, durch die er der abstrakten sozialistisch-kommunistischen Überzeugung, die er aus Deutschland mitgebracht hatte, eine völlig neue, eine "materialistische» Grundlage gab. Mit Max Weber (1922: 214) gesprochen: Die kommunistischen Gestirne, an denen sich Engels orientierte, warfen Licht auf ein für die deutsche Linke fast völlig unbekanntes Land und Engels vermochte es, daraus ein neues Ganzes zu entwerfen, das zugleich vieles Einzelne im neuen Licht zeigte.

Engels hat das Anliegen seines Buches, mitten im Verfassen des Werkes, in einem Brief an Marx vom Oktober 1844 zum Ausdruck gebracht: »Ich war in Köln drei Tage und erstaunte über die ungeheure Propaganda, die wir dort gemacht haben. Die Leute sind sehr tätig, aber der Mangel an einem gehörigen Rückhalt ist doch sehr fühlbar. Solange nicht die Prinzipien logisch und historisch aus der bisherigen Anschauungsweise und der bisherigen Geschichte und als die notwendige Fortsetzung derselben in ein paar Schriften entwickelt sind, solange ist es doch alles noch halbes Dösen und bei den meisten blindes Umhertappen." (Engels 1844: 5) Eben dies leistete sein 
Werk. Deshalb hatte Franz Mehring völlig recht, als er diese engelssche Schrift und nicht eine Arbeit von Marx aus jener Zeit als »die erste große Urkunde des wissenschaftlichen Sozialismus« (Mehring 1905: 553) bezeichnete. Stolz blickte Engels selbst 1872 in seiner Artikelsammlung Zur Wohnungsfrage zurück auf sein Buch von 1845 und schrieb, dass es sein Ziel gewesen sei, "dem damals entstehnden, in hohlen Phrasen herumfahrenden deutschen Sozialismus eine tatsächliche Unterlage zu geben durch Beschreibung der von der modernen großen Industrie geschaffnen Gesellschaftszustände« (Engels 1872: 285). ${ }^{3}$

Engels' Werk Die Lage der arbeitenden Klasse in England ist deshalb die Gründungsurkunde des wissenschaftlichen Sozialismus, weil Engels als Erster auf der Höhe der Sozialwissenschaften und Philosophie seiner Zeit aus der detaillierten Analyse der geschichtlichen Entstehung und Entwicklung der bürgerlich-kapitalistischen Gesellschaft, der Herausbildung der modernen Arbeiter*innenklasse und ihrer ökonomischen, sozialen und kulturellen Lage konkrete strategische Schlussfolgerungen mit sozialistischer und kommunistischer Zielstellung begründet. Von Engels kann auch heute noch gelernt werden, wie dies gemacht

3 Wenn heute gefordert wird »System Change, not Climate Change«, so braucht es auch dafür Erzählungen, die diesem Ziel eine Unterlage geben. Aus der Vielzahl der Beispiele sei nur auf einige verwiesen (Dellheim u.a. 2012; Klein 2013; Brand/Wissen 2017; Winker 2015; Dörre 2018; Jugendrat der Generationenstiftung 2019; Kaufmann u.a. 2019). wird, auch wenn die realen wie wissenschaftlichen Bedingungen sich wesentlich gewandelt haben.

Engels' Schrift ist erstens aus der Position teilnehmender Beobachtung geschrieben. Dies macht er in seiner Widmung »An die arbeitenden Klassen Großbritanniens « deutlich:»[...] mir war es um mehr zu tun als um die nur abstrakte Kenntnis meines Gegenstandes, ich wollte euch in euren Behausungen sehen, euch in eurem täglichen Leben beobachten, mit euch plaudern über eure Lebensbedingungen und Schmerzen, Zeuge sein eurer Kämpfe gegen die soziale und politische Macht eurer Unterdrücker « (Engels 1845: 229). Er hat die Versammlungen der Sozialist*innen wie Chartist*innen besucht (vgl. Engels 1843), wirkte als Redakteur ihrer wichtigsten Zeitungen, besuchte - auch mit seiner Partnerin Mary Burns - die Arbeiter*innen zu Hause.

Zweitens ist es bemerkenswert, wie reflektiert Engels mit den Quellen umgeht und vor allem auf die Berichte der von Regierungsseite eingesetzten Inspektoren oder auch der politischen Gegner zurückgreift, um Objektivität zu sichern. Er will die Fakten für sich selbst sprechen lassen, macht immer wieder auf gegenwirkende Faktoren aufmerksam. Weder idealisiert, noch verteufelt er. Die Ursachen sieht er vor allem in den Verhältnissen selbst, denen die Akteure unterworfen sind. Indem er aber von unten auf das Ganze blickt, sieht er das Ganze nicht in seiner Gestalt des herrschaftlichen Scheins, sondern ausgehend von den Produktions- und Reproduktionsverhältnissen, deren in die Fabriken verdammten, in die Hinterhäuser gezwängten »Heldinnen und 
Helden «, um an die aktuelle CoronaSprache anzuknüpfen. Man kann mit dem Blick von heute auch Voreingenommenheiten bei Engels erkennen, wenn die Ir*innen geschildert werden, wenn patriarchale Stereotype reproduziert werden - immer wieder relativiert, aber unübersehbar.

Drittens sei auf die Komposition des Werkes hingewiesen. Es beginnt mit einer klaren These, die dann sozialwissenschaftlich empirisch begründet wird, und Engels schließt mit einer eindeutigen strategischen Orientierung und Perspektive. Die These wird am Beginn der "Vorrede « in nüchternster Sprache vorgestellt: »Die Lage der arbeitenden Klasse ist der tatsächliche Boden und Ausgangspunkt aller sozialen Bewegungen der Gegenwart, weil sie die höchste, unverhüllteste Spitze unsrer bestehenden sozialen Misere ist. Der französische und deutsche Arbeiterkommunismus sind direkt, der Fourierismus und der englische Sozialismus sowie der Kommunismus der deutschen gebildeten Bourgeoisie sind indirekt durch sie erzeugt. Einerseits, um den sozialistischen Theorien, andrerseits, um den Urteilen über ihre Berechtigung einen festen Boden zu geben, um allen Schwärmereien und Phantastereien pro et contra ein Ende zu machen, ist die Erkenntnis der proletarischen Zustände deshalb eine unumgängliche Notwendigkeit. Die proletarischen Zustände existieren aber in ihrer klassischen Form, in ihrer Vollendung nur im britischen Reich, namentlich im eigentlichen England; und zugleich ist nur in England das nötige Material so vollständig zusammengetragen und durch offizielle Untersuchungen konstatiert, als es zu einer irgendwie erschöpfenden Darstellung des Gegenstandes nötig ist." (Engels 1845: 232)

Auf den folgenden 250 Druckseiten werden die Entstehung des Proletariats infolge der industriellen Revolution unter bürgerlichen Verhältnissen, seine grundlegenden Merkmals, seine Lebensweise in den Städten des Industriezeitalters, die einzelnen Gruppen des Proletariats in ihrer Differenzierung und Gegensätzlichkeit entlang von Qualifikation, Geschlecht, Ethnie (»irische Frage«), Organisationsmacht, Bewusstheit und Erfahrung dargestellt.

Engels verliert sich aber nicht in den Einzelheiten, sondern er verbindet sie immer wieder mit klar formulierten Grundeinsichten. Deshalb kann er anschaulich den Klassencharakter der Städte rekonstruieren bis zu den frühen Tendenzen der Gentrifizierung. Er zeigt auf, wie die spontanen Tendenzen der entfesselten Marktkräfte die Not, das Elend hinter den Fassaden respektabler Straßen unsichtbar machen, wie Umweltzerstörung, Epidemien, Luftverschmutzung vor allem die untersten Gruppen der Gesellschaft treffen. So ist auch heute, wenn die Corona-Pandemie vor allem nach den Kriterien von Klasse, Ethnie und Geschlecht zuschlägt - in den einzelnen Ländern und global.

Er entschlüsselt auch die Hauptursache der strukturellen Schwäche des Proletariats gegenüber der Bourgeoisie ihre existentielle Konkurrenz miteinander auf dem Arbeitsmarkt. Und er zeigt, dass die Stellung als Proletarier*innen, so privilegiert Einzelne oder eine Gruppe auch sein mag, immer bedeutet, auf unsicherem sozialen Boden, unter den 
Bedingungen latenter Prekarität $\mathrm{zu}$ leben, wie jüngst selbst die Spitze der Arbeiteraristokratie, die Piloten von Lufthansa, erfuhren. Die outgesourcte Reinigungskraft, die Pfleger*innen, das Prekariat der Lieferdienste wusste es längst. Von Engels kann gelernt werden, dass man sich eben nicht auf die Symptome des nackten Elends konzentrieren dürfen, denn "noch viel demoralisierender als die Armut wirkt auf die englischen Arbeiter die Unsicherheit der Lebensstellung, die Notwendigkeit, vom Lohn aus der Hand in den Mund zu leben, kurz das, was sie zu Proletariern macht. (Ebd.: 343) Die Ursache für diese Prekarität proletarischer Existenz ist für Engels die Konkurrenz der Arbeiter*innen miteinander unter der Bedingung, dass nicht sie, sondern die Bourgeoisie über die Produktionsbedingungen des Lebens verfügen: »Diese Konkurrenz der Arbeiter gegeneinander ist [...] die schlimmste Seite der jetzigen Verhältnisse für den Arbeiter, die schärfste Waffe gegen das Proletariat in den Händen der Bourgeoisie.« (Ebd.: 306f.)

Und schließlich zeichnet Engels die Entstehung einer selbstbewussten Arbeiter*innenbewegung in ihre Etappen nach - "the making of the working class by the working class «. Als wichtigste strategische Aufgabe sieht Engels die Verbindung und schließlich Vereinigung von sozialistischer Bewegung der Owenit*innen und demokratischer Massenbewegung der Chartist*innen. Erst dann wenn die sozialistischen Einsichten und die proletarische Massenbewegung eine Einheit bildet, so Engels, »wird die Arbeiterklasse wirklich die Herrscherin von England sein« (ebd.: 453). Sein
Werk schließt der 24-Jährige mit der Prophezeiung: »Der Krieg der Armen gegen die Reichen, der jetzt schon im einzelnen und indirekt geführt wird, wird auch im allgemeinen, im ganzen und direkt in England geführt werden. Es ist zu spät zur friedlichen Lösung. Die Klassen sondern sich schroffer und schroffer, der Geist des Widerstandes durchdringt die Arbeiter mehr und mehr, die Erbitterung steigt, die einzelnen Guerillascharmützel konzentrieren sich zu bedeutenderen Gefechten und Demonstrationen, und ein kleiner Anstoß wird bald hinreichen, um die Lawine in Bewegung zu setzen. Dann wird allerdings der Schlachtruf durch das Land schallen: `Krieg den Palästen, Friede den Hütten! - - dann wird es aber zu spät sein, als dass sich die Reichen noch in acht nehmen könnten.« (Ebd.: 506)

$\mathrm{Zu}$ Engels' 200. Geburtstag sollte man Marx folgen und Engels' Werk Die Lage der arbeitenden Klasse in England endlich nicht nur erneut lesen, sondern gründlich studieren und lernen, wie weltgeschichtlich eingreifende Anschauungen entstehen. Marx hatte das Buch während seiner Arbeit am Kapital - Engels musste sich immer noch dem "Schacher « unterwerfen, um Marx unterstützen zu können - 1863 neu zur Hand genommen und an sein Alter Ego geschrieben: »Das Wiederlesen Deiner Schrift hat mich mit Bedauern das Altern merken lassen. Wie frisch, leidenschaftlich, kühn vorausgreifend und ohne gelehrte und wissenschaftliche Bedenken wird hier noch die Sache gefasst! Und die Illusion selbst, dass morgen oder übermorgen das Resultat auch geschichtlich ans Tageslicht 
springen wird, gibt dem Ganzen eine Wärme und lebenslustigen Humor wogegen das spätere `Grau in Grauk verdammt unangenehm absticht." (Marx 1863: 343) Dem ist nichts hinzuzufügen.

- Happy birthday, Friedrich Engels! -

\section{Literatur}

Bourdieu, Pierre (1990): Was heißt sprechen? Die Ökonomie des sprachlichen Tausches. Wien.

Brand, Ulrich / Wissen, Markus (2017): Imperiale Lebensweise: Zur Ausbeutung von Mensch und Natur in Zeiten des globalen Kapitalismus. München.

Brie, Michael (2020): Sozialist-Werden. Friedrich Engels in Manchester und Barmen 1842-1845. Berlin. URL: https://www.rosalux.de, Zugriff: 30.6.2020.

Carver, Terrell (2020): Engels before Marx. London.

Dellheim, Judith / Brangsch, Lutz / Wolf, Frieder Otto / Spangenberg, Joachim (2012): Den Krisen entkommen. Sozialökologische Transformation. Berlin.

Dörre, Klaus (2018): Neo-Sozialismus oder: Acht Thesen zu einer überfälligen Diskussion. In: Blätter für deutsche und internationale Politik, Nr. 6: 105-115.

Engels, Friedrich (1843): Briefe aus London. In: Marx-Engels-Werke, Bd. 1. Berlin: 468-479.

- (1844): Brief an Karl Marx, Anfang Oktober 1844. In: Marx-Engels-Werke, Bd. 27. Berlin: 5-8.

- (1845): Die Lage der arbeitenden Klasse in England. Nach eigner Anschauung und authentischen Quellen. In: Marx-EngelsWerke, Bd. 2. Berlin: 225-506.

- (1872): Zur Wohnungsfrage. In: MarxEngels-Werke, Bd. 18. Berlin: 209-287.

- (1884): Brief an Johann Philipp Becker, 15. Oktober 1884. In: Marx-Engels-Werke, Bd. 36. Berlin: 218-219.
Heß, Moses (1843): Brief an Berthold Auerbach vom 19. Juni 1843. In: Silberner, Edmund (Hg): Moses Heß Briefwechsel. Gravenhage 1959: 103.

Jugendrat der Generationenstiftung (2019): Ihr habt keinen Plan, darum machen wir einen! 10 Bedingungen für die Rettung unserer Zukunft. München.

Kaufmann, Sina Kamala / Timmermann, Michael / Botzki, Annemarie (Hg.) (2019): Wann wenn nicht wir*. Ein Extinction Rebellion Handbuch. Frankfurt/M.

Klein, Dieter (2013): Das Morgen tanzt im Heute. Transformation im Kapitalismus und über ihn hinaus. Hamburg. URL: https:// www.rosalux.de, Zugriff: 30.6.2020.

Krätke, Michael (2020): Friedrich Engels oder: Wie ein Cotton-Lord den Marxismus erfand. Berlin.

Lucas, Rainer / Pfriem, Reinhard / Westhoff, Hans-Dieter (Hg.) (2020): Arbeiten am Widerspruch - Friedrich Engels zum 200. Geburtstag. Marburg.

Marx, Karl (1863): Brief an Friedrich Engels, 9. April 1863. In: Marx-Engels-Werke, Bd. 30. Berlin: 340-343.

Mehring, Franz (1905): Friedrich Engels. In: Die Neue Zeit, 23.2(44): 553-555.

Marx-Engels-Werke (MEW), Marx, Karl / Engels, Friedrich (1956ff.): Marx-EngelsWerke. Berlin.

Puckett, Kent (2016): Narrative Theory. A Critical Introduction. Cambridge.

Röhrig, Tilman (2019): Und morgen eine neue Welt. Der große Friedrich-Engels-Roman. München.

Ullrich, Horst (1966): Der junge Engels. Eine historisch-biographische Studie seiner weltanschaulichen Entwicklung in den Jahren 1843 bis 1845 . Zweiter Teil. Berlin.

Weber, Max (1922): Die Objektivität sozialwissenschaftlicher und sozialpolitischer Erkenntnis. In: Gesammelte Aufsätze zur Wissenschaftslehre. Tübingen: 146-214.

Winker, Gabriele (2015): Care Revolution. Schritte in eine solidarische Gesellschaft. Bielefeld. 